\title{
Power-Aware DBMS: Potential and Challenges
}

\author{
Yi-cheng $\mathrm{Tu}^{1}$, Xiaorui $\mathrm{Wang}^{2}$, and Zichen $\mathrm{Xu}^{1}$ \\ 1 Department of Computer Science and Engineering \\ 4202 E. Fowler Ave., ENB118 \\ Tampa, FL 33620 \\ 2 Department of Electrical Engineering and Computer Science \\ The University of Tennessee \\ Knoxville, TN 37996 Univ. of Tennessee \\ \{ytu,zxu5\}@mail.usf.edu, xwang@eecs.utk.edu
}

\begin{abstract}
Energy consumption has become a first-class optimization goal in computing system design and implementation. Database systems, being a major consumer of computing resources (thus energy) in modern data centers, also face the challenges of going green. In this position paper, we describe our vision on this new direction of database system research, and report the results of our recent work on this topic. We describe our ideas on the key issues in designing a power-aware DBMS and sketch our solutions to such issues. Specifically, we believe that the ability for the DBMS to dynamically adjust various knobs to satisfy energy (and performance) goals is the main technical challenge in this paradigm. To address that challenge, we propose dynamic modeling and tuning techniques based on formal feedback control theory. Our preliminary data clearly show that the energy savings can be significant.
\end{abstract}

Keywords: Power-aware DBMS, feedback control, energy profile identification, system modeling, power cost estimation.

\section{Introduction}

The steep increase of energy consumption of computers have made power management a critical issue in system design and implementation. As shown in our previous work [2, a basic design of P-DBMS introduces multiple control knobs that enable real-time adjustment of system behavior. A typical database system bears many uncertainties in its workload and environment therefore the problem of DBMS control for energy-saving purposes cannot be mapped into a conventional optimization problem. Our proposal to tackle this problem is to view it as an optimal control problem and utilize rigorous control-theoretical analysis and system design techniques to accomplish energy saving and performance goals. Our control-based solution, in contrast to ad hoc heuristics that are widely used in solving similar tuning problems, has the advantage of providing guaranteed performance and resistance to system/environmental dynamics. 


\section{Overview of P-DBMS Design}

Our vision of building an energy-efficient DBMS is to enhance current DBMS components with energy-related functionalities, rather than building these components from scratch. This allows us to minimize the impacts on the current DBMS architecture that is well-designed for performance-driven query processing. The design goal of the system is as follows:

Problem 1. Given a performance bound, the power consumption of the database system should be minimized.

The above design goal reflects the idea that performance is the most critical issue, while saving energy cost is a best-effort requirement based on a tolerance level of performance degradation.

\section{Feedback Control for Power Optimization}

Traditionally, solutions to adaptive power management problems, as well as those in self-tuning databases, heavily rely on heuristics. Recently, however, feedback control theory has been successfully applied to power control in servers and database tuning [1]. The benefit of having control theory as a theoretical foundation is that we can have (1) standard approaches to choosing the right control parameters so that exhaustive iterations of tuning and testing are avoided; (2) theoretically guaranteed control performance such as accuracy, stability, short settling time, and small overshoot; and (3) quantitative control analysis when the system is suffering unpredictable workload variations. This rigorous design methodology is in sharp contrast to heuristic-based adaptive solutions that rely on extensive empirical evaluation and manual tuning.

Intuitively, the power mode of hardware should be set to a level such that the system performance converges to the tolerance bound mentioned in PROBLEM 1. This is because making the performance better than the bound is not necessary and also implies less energy saving. We are developing a feedback control loop to satisfy the runtime power and performance requirements set in PROBLEM 1.

\section{Conclusions}

In this paper, we elaborated on the issues in building such a PDBMS system and how such issues can be resolved. Our idea was to utilize formal feedback control theory to achieve effective modeling and system control. We conclude that power-aware DBMS is a meaningful and interesting approach for tackling the problem of energy-efficient database systems.

\section{References}

1. Lightstone, S., et al.: Control theory: a foundational technique for self managing databases. In: ICDE Workshops, pp. 395-403 (2007)

2. $\mathrm{Xu}, \mathrm{Z}$., Tu, Y., Wang, X.: Exploring power- performance tradeoffs in database systems. In: Proceedings of ICDE (2010) 